\title{
Circular Loop Antennas Reactively Loaded for a Uniform Traveling-Wave Current Distribution
}

\author{
R. L. Li, J. Laskar, and M. M. Tentzeris \\ School of Electrical and Computer Engineering \\ Georgia Institute of Technology, Atlanta, GA 30332-0250, USA
}

\begin{abstract}
In this paper, it is demonstrated that a uniform traveling-wave current distribution on a circular loop of one-wavelength circumference can be obtained by loading the loop with a lumped reactance at a position of $45^{\circ}$ away from the feed point. To achieve a uniform traveling-wave current distribution, the loading reactance and the input impedances of the loaded and unloaded loop antennas need to satisfy certain relations from which the loading reactance can be predicted theoretically. It is shown that the circular loop antenna with uniform traveling-wave current distribution results in a lower profile, a more symmetrical circularly polarized radiation pattern, and a much better input impedance matching.
\end{abstract}

\section{INTRODUCTION}

It is well known that a single-fed circular loop antenna of one-wavelength circumference (called one-wavelength loop in short) creates a standing-wave current distribution and thus radiates a linearly polarized wave in the far-field zone [1]-[3]. It is also easy to understand that a onewavelength circular loop can radiate perfectly circularly polarized waves in the direction perpendicular to the plane of the loop if a uniform traveling-wave current distribution is generated [4]. A common way to produce a uniform traveling-wave current distribution is to feed the loop antenna with two $90^{\circ}$ phase shifted generators which are located $90^{\circ}$ [5]. In 1980s, it was demonstrated that a traveling-wave current distribution could be obtained if a circular loop is reactively loaded at a position of $45^{\circ}$ away from the feed point [6]. However, the traveling-wave current distributions obtained were far from uniform due to a high-value ( $>300 \mathrm{ohms}$ ) reactance used. Most importantly, no theoretical prediction was given in [6] for the value of the loading reactance; therefore it was not clear why a traveling-wave current distribution could be achieved by the reactive load. It is our purpose to demonstrate that it is possible to theoretically predict the loading reactance for the launching of a uniform traveling-wave current distribution along a onewavelength circular loop antenna. It will be proved that there are simple relations among the loading reactance and the input impedances of the loaded and unloaded loop antennas. Also, it will be found that the uniform traveling-wave current distribution can be created when a onewavelength circular loop is situated at a height of much less than a quarter-wavelength (about 0.05-0.15 wavelengths considered in this paper) above the ground plane. Thus, the low-profile circular loop antenna can be loaded with a low-value capacitive reactance, leading to smaller input impedance which would facilitate the matching of the antenna.

\section{PREDICTION OF REACTANCE LOADING FOR A UNIFORM TRAVELING- WAVE CURRENT DISTRIBUTION}

As shown in Fig. 1, consider a circular wire loop antenna above ground with a loop radius of $b$ and a wire radius of $a$. It is assumed that the circumference of the loop is one wavelength $\left(\lambda_{0}\right)$. The loop is fed by a voltage source $\mathrm{V}_{0}$ at $\phi=0$ and is loaded by a lumped impedance $\mathrm{Z}_{\mathrm{L}}$ (a capacitive loading) at $\phi=\pi / 4$. Under the "thin wire" approximation, the current distribution on the loop can be represented by a filamentary current $i(\phi, t)=I(\phi) e^{j \omega t}$ along the loop circumferential axis. We assume a uniform travelingwave current distribution created on the loop such that

$$
I(\phi)=I_{0} e^{ \pm j \phi} \text {. }
$$


Applying the compensation theorem, we can replace the load $\mathrm{Z}_{\mathrm{L}}$ with an equivalent loading voltage $\mathrm{V}_{\mathrm{L}}$ :

$$
V_{L}=-Z_{L} I(\pi / 4)=-Z_{L} I_{0} e^{ \pm j \pi / 4} .
$$

According to the principle of superposition, the current distribution $I(\phi)$ can be obtained by the superposition of the two currents in the loop when fed by each voltage source alone, that is,

$$
I(\phi)=I^{0}(\phi)+I^{L}(\phi),
$$

where $I^{0}(\phi)$ and $I^{L}(\phi)$ are generated by the feeding voltage $\mathrm{V}_{0}$ at $\phi=0$ and the loading voltage $\mathrm{V}_{\mathrm{L}}$ at $\phi=\pi / 4$, respectively. It is a reasonable approximation to represent these current distributions on a single-fed one-wavelength circular loop with a cosinusoidal variation:

$$
I^{0}(\phi)=I_{0}^{0} \cos (\phi), I^{L}(\phi)=I_{0}^{L} \cos (\phi-\pi / 4) .
$$

With the input impedance of the circular loop antenna without the load, $Z_{\text {in }}^{U}$ (simply called "unloaded input impedance”), the current constants $I_{0}^{0}$ and $I_{0}^{L}$ are given by

$$
I_{0}^{0}=V_{0} / Z_{\text {in }}^{U}, I_{0}^{L}=V_{L} / Z_{\text {in }}^{U}=-Z_{L} I_{0} e^{ \pm j \pi / 4} / Z_{\text {in }}^{U} .
$$

Substituting (4) in (3) with $I_{0}^{0}$ and $I_{0}^{L}$ from (5) leads to

$$
I(\phi)=\frac{V_{0}}{Z_{i n}^{U}} \cos \phi-I_{0} e^{ \pm j \pi / 4} \frac{Z_{L}}{Z_{\text {in }}^{U}} \cos (\phi-\pi / 4) .
$$

Substituting $I(\phi)=I_{0} e^{ \pm j \phi}$ into the left side of (6) and replacing $I_{0}$ with $I_{0}=V_{0} / Z_{\text {in }}^{L}$, where $Z_{i n}^{L}$ is the input impedance of the circular loop antenna with the load (simply called "loaded input impedance"), we obtain

$$
\frac{V_{0}}{Z_{\text {in }}^{L}} e^{ \pm j \phi}=\frac{V_{0}}{Z_{\text {in }}^{U}} \cos \phi-\frac{V_{0}}{Z_{\text {in }}^{L}} \frac{Z_{L}}{Z_{\text {in }}^{U}} e^{ \pm j \pi / 4} \cos (\phi-\pi / 4) .
$$

After a simple algebra manipulation, (7) becomes

$$
Z_{L}=2 Z_{i n}^{U} \frac{\left(Z_{i n}^{L} / Z_{i n}^{U}-1\right) \cos \phi \mp j \sin \phi}{(1 \pm j)(\cos \phi+\sin \phi)} .
$$

Since $Z_{L}$ (fixed at $\phi=\pi / 4$ ) is independent of $\phi$, we must have

$$
\left(Z_{\text {in }}^{L} / Z_{\text {in }}^{U}-1\right)=\mp j \text {. }
$$

This is the condition for a uniform traveling-wave current distribution on the onewavelength circular loop. Under the condition, we can find by substituting (9) in (8) the value of the loading impedance $Z_{L}$ :

$$
Z_{L}=-(1 \pm j) Z_{\text {in }}^{U} \text { or } Z_{L}=\mp j Z_{i n}^{L},
$$

which implies that the loading impedance can be predicted from the unloaded input impedance.

\section{AN EXPAMPLE OF THE REALIZATION OF A UNIFORM TRAVELING-WAVE CURRENT DISTRIBUTION}

Consider a circular loop above ground plane with two different heights: $h=0.05 \lambda_{0}$ and $h=0.08 \lambda_{0}$. Fig. 2 shows the simulated frequency characteristics of the unloaded input impedance. From this figure, we can indeed find for each height an intersection point where the input impedance $Z_{i n}^{U}=R_{i n}^{U}+j X_{\text {in }}^{U}$ with $R_{i n}^{U}=X_{\text {in }}^{U}$ and the frequency is close 
to $f_{0}=\mathrm{c} / \lambda_{0}\left(f<1.1 f_{0}\right)$. Since for all frequencies and heights of interest, the real part and the imaginary part of the unloaded impedance have the same sign, the loading has to be capacitive. It also can be predicted that a left-hand uniform traveling-wave current distribution may probably be obtained at $f=f_{i p}$ if the circular loop is loaded at $\phi=\pi / 4$ with a capacitive reactance $Z_{L}=-j 2 R_{i p}$. To verify the prediction, we now load the circular loop with $Z_{\mathrm{L}}=-\mathrm{j} 27$ and $-\mathrm{j} 78$ ohms for $h=0.05 \lambda_{0}$ and $h=0.08 \lambda_{0}$, respectively, and draw the simulated current distributions at $f=f_{i p}$ in Fig. 3 . As expected, these current distributions do show left-hand uniform traveling-wave features, particularly for $h=0.05 \lambda_{0}$ where the magnitude of the current is almost constant and the phase varies with $\phi$ linearly. The performance of circular polarization of the capacitance-loaded circular loop is shown in Fig. 4. We can see a good axial ratio $(<3 \mathrm{~dB})$ around $f_{i p}$. On the basis of theoretical prediction, the input impedance of the circular loop antenna loaded with the capacitive reactance can be obtained, as shown in Fig. 5. It is observed that the values of both the input resistance and the input reactance increase as the loop height and the wire thickness increase. For a VSWR (voltage standing-wave ratio) of less than 2 (in a 50-ohms feeding system), the loop height should be less than $0.1 \lambda_{0}$.

\section{CONCLUSION}

It has been demonstrated that it is possible to theoretically predict the values of the reactive loading of a circular loop with one-wavelength circumference for uniform traveling-wave current distribution. For a reactive load located on the circular loop at a position of $45^{\circ}$ away from the feed point, the value of the loading reactance has to be equal to two times of the input resistance of the unloaded circular loop antenna. It is also found that to achieve a uniform traveling-wave current distribution, the unloaded input resistance and input reactance must be equal in value and that the loaded loop antenna should have a purely resistive input impedance with a value also equal to two times of the unloaded input resistance. A uniform traveling-current distribution has been achieved by loading the loop above a ground plane with a capacitance. The capacitance-loaded circular loop antennas with uniform traveling-wave current distribution have a lower profile, more symmetrical radiation pattern, and much better input impedance matching.

\section{Acknowledgement}

The authors wish to acknowledge the support of Georgia Electronic Design Center, the NSF CAREER Award under contract NSF \#9964761, the NSF Award NSF ECS-0313951, and the NSF Packaging Research Center,

\section{References}

[1] T. T. Wu, "Theory of thin circular loop antennas,"J. Math. Phys. vol. 3, pp.1301-1304, 1962.

[2] B. R. Rao, "Far field patterns of large circular loop antennas: Theoretical and experimental results," IEEE Trans. Antenna and Propagat., vol. 16, pp. 269-270, Mar. 1968.

[3] W. L. Stutzman and G. A. Thiele, Antenna Theory and Design, John Wiley \& Sons, Inc., pp. 205-210, 1998.

[4] R. S. Elliott, Antenna Theory and Design, IEEE Press, John Wiley \& Sons, Inc., pp. 71-73, 2003.

[5] H. Nakano, N. Tsuchiya, T. Suzuki, and J. Yamauchi "Loop and spiral line antennas at microstrip substrate surface," Proceedings of the Sixth International Conference on Antenna and Propagation (ICAP), pp. 196-200, 1989.

[6] S. Okuba and S. Tokumaru "Reactively loaded loop antennas with reflectors for circular polarization," The Transactions of the IECE of Japan, vol. J65-B, pp. 1044-1051, Aug. 1982. 


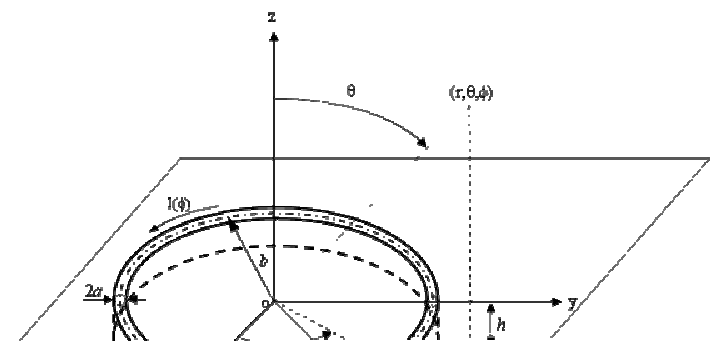

